\title{
Arsenic Removal and Recovery of Germanium and Tungsten in Toxic Coal Fly Ash from Lignite by Vacuum Distillation with Sulfurizing Reagent
}

\author{
Lingen Zhang, Qingming Song and Zhenming Xu * \\ School of Environmental Science and Engineering, Shanghai Jiao Tong University, \\ 800 Dongchuan Road, Shanghai 200240, People's Republic of China
}

\section{Supporting Information Content}

11 Pages (including the cover page)

4 Figures

5 Tables

Figure S1. Schematic illustration of the vacuum quartz tube furnace;

Figure S2. Morphology and EDS analysis of As and S element;

Figure S3. Recovery pathways and potential applications of As, W and Ge;

Figure S4. Kinetics curve of typical mechanism functions;

Table S1. Basic component and element analysis of lignite in the study

Table S2. Chemical components of coal fly ash (unit: wt. \%)

Table S3. Removal ratio of As and content of Ge and $\mathrm{W}$ in residue under optimal conditions $(550 \mathrm{oC}$ of reaction temperature, adding $50 \mathrm{wt} \% \%$ sulfurizing agent, system pressure of $1 \mathrm{~Pa}$, and 240 min of reaction time) 
Table S4. Chemical components of residues from coal fly ash (WL1-1, WL1-2..., WL4-4) (unit: wt. \%)

Table S5. Mechanism functions of kinetics and fitting degree for the vacuum reaction 


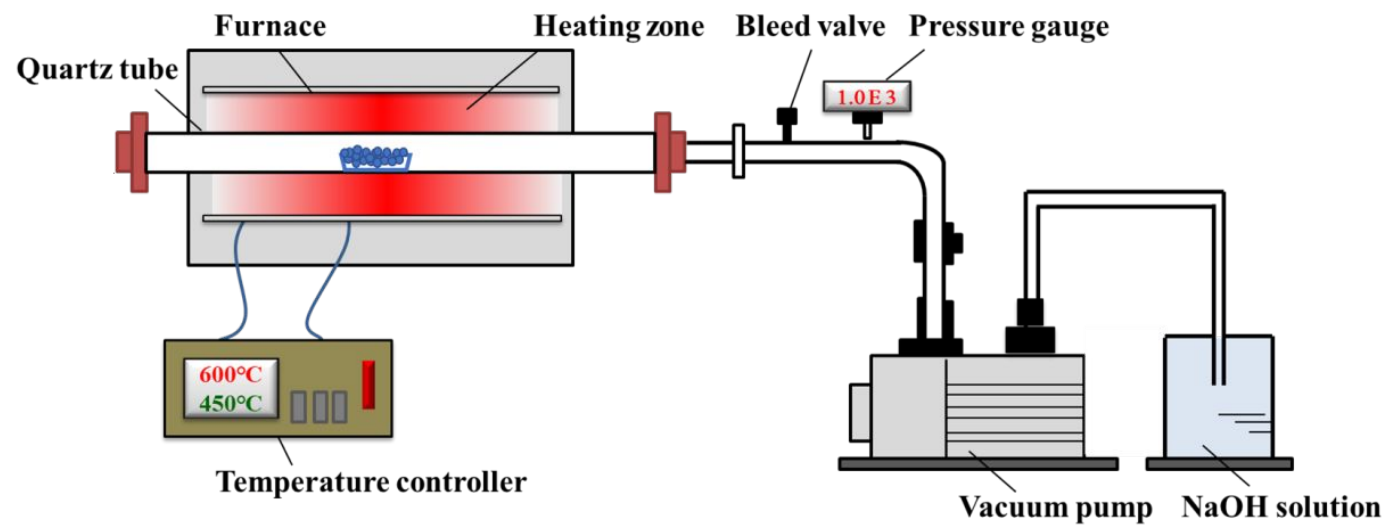

Figure S1. Schematic illustration of the vacuum quartz tube furnace 


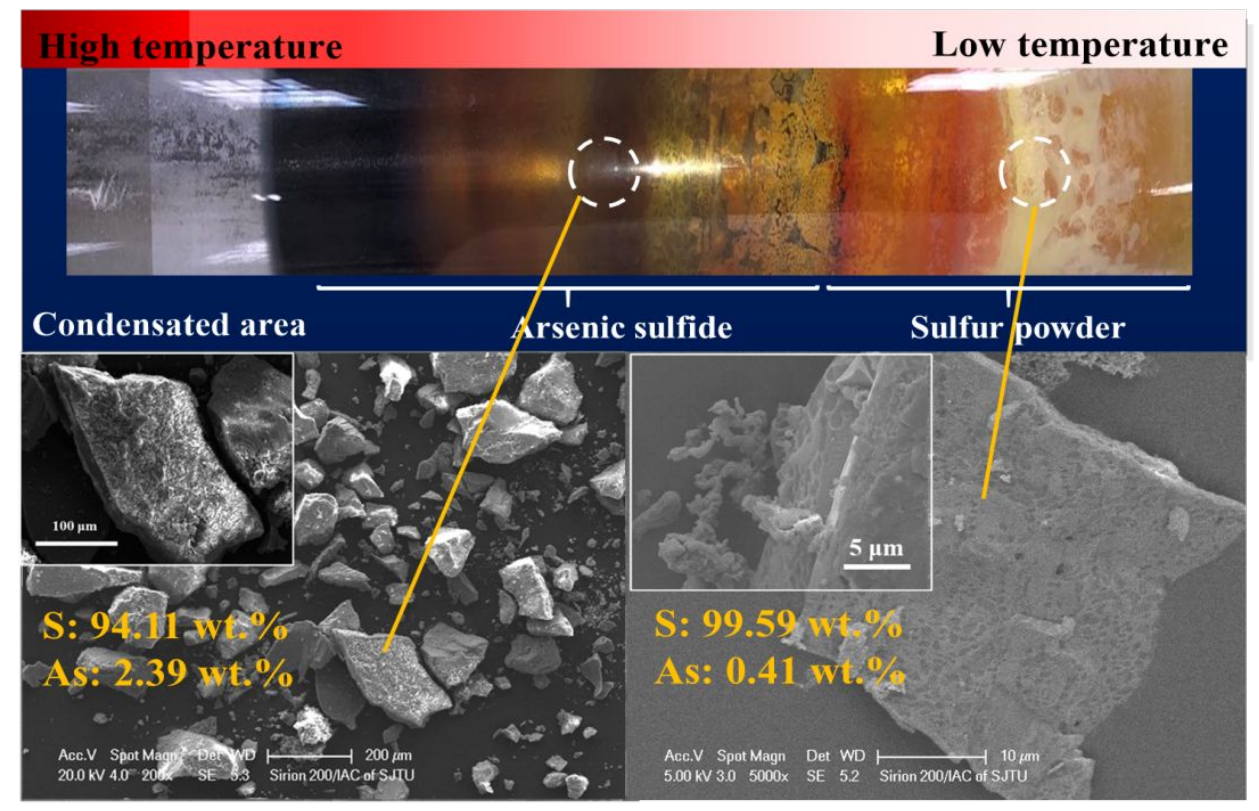

Figure S2. Morphology and EDS analysis of As and S element 


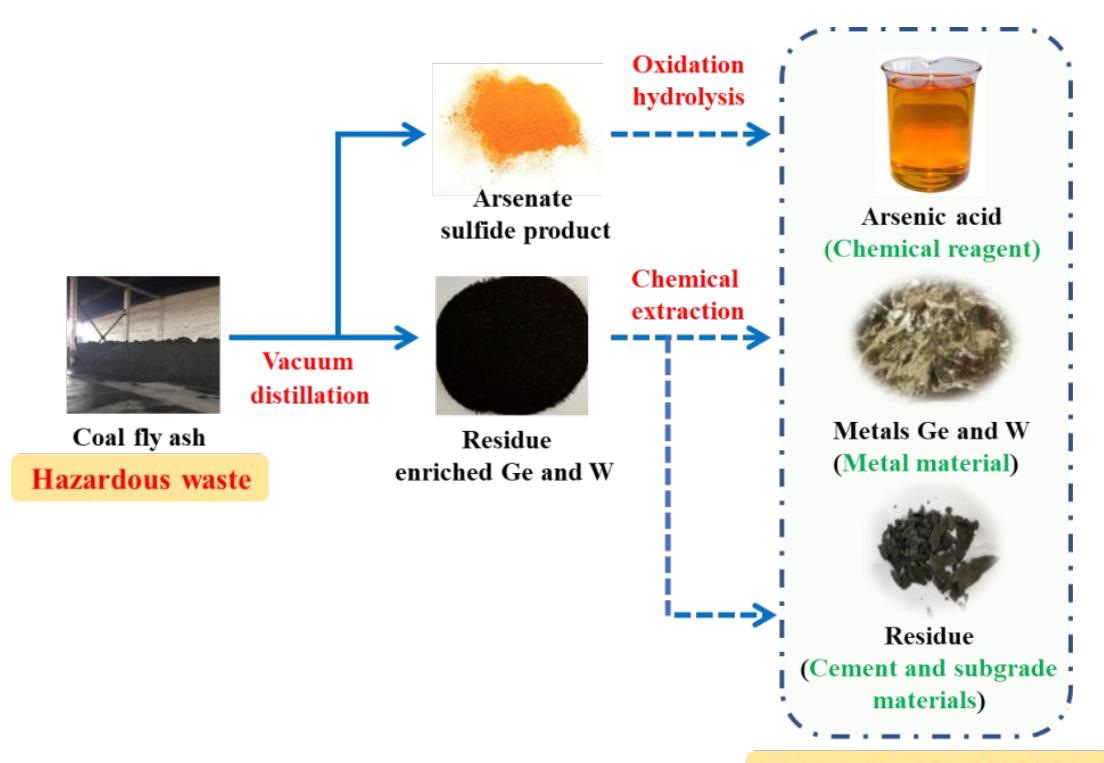

Future recycled products

Figure S3. Recovery pathways and potential applications of As, W and Ge 


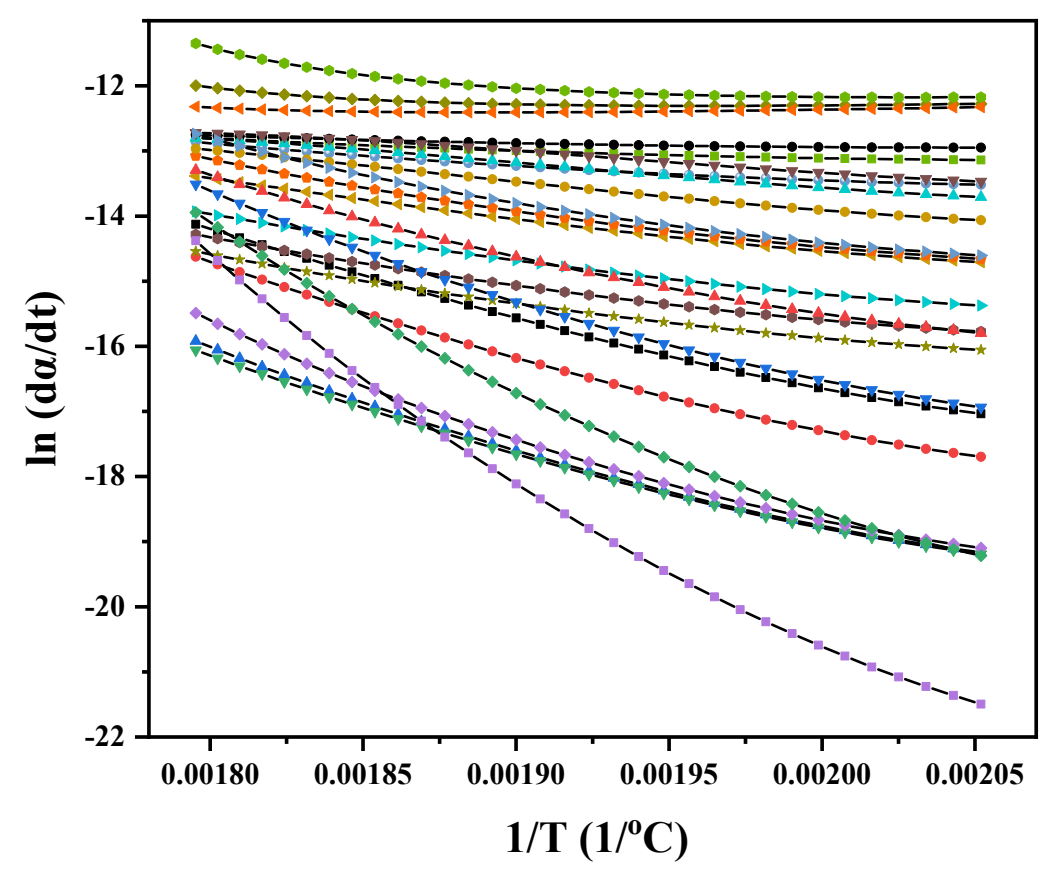

Figure S4. Kinetics curve of typical mechanism functions 
Table S1. Basic component and element analysis of lignite in the study

\begin{tabular}{|c|c|c|c|c|c|c|}
\hline $\begin{array}{c}\text { Basic } \\
\text { component } \\
\text { analysis }\end{array}$ & $M_{t}(\%)$ & $M_{a d}(\%)$ & $\mathbf{A}_{\mathrm{d}}(\%)$ & $V_{\text {daf }}(\%)$ & $F_{c d}(\%)$ & $\begin{array}{c}\text { Calorific } \\
\text { value (Q, } \\
\text { KJ/kg) }\end{array}$ \\
\hline WL-1 & 22.62 & 3.12 & 21.63 & 36.71 & 38.2 & 19.37 \\
\hline WL-2 & 20.21 & 4.31 & 23.82 & 34.35 & 37.46 & 21.03 \\
\hline WL-3 & 21.74 & 3.7 & 21.92 & 37.1 & 36.43 & 21.85 \\
\hline WL-4 & 18.43 & 2.8 & 22.43 & 35.65 & 38.7 & 22.78 \\
\hline $\begin{array}{l}\text { Element } \\
\text { analysis }\end{array}$ & $\mathrm{C}(\%)$ & \multicolumn{2}{|c|}{ H (\%) } & N (\%) & O (\%) & S (\%) \\
\hline WL-1 & 40.96 & \multicolumn{2}{|c|}{2.58} & 0.61 & 11.47 & 0.45 \\
\hline WL-2 & 59.6 & \multicolumn{2}{|c|}{3.8} & 0.81 & 19.6 & 1.26 \\
\hline WL-3 & 64.6 & \multicolumn{2}{|c|}{3.85} & 0.93 & 17.4 & 1.73 \\
\hline WL-4 & 60.3 & \multicolumn{2}{|c|}{3.92} & 0.9 & 20.8 & 1.3 \\
\hline
\end{tabular}


Table S2. Chemical components of coal fly ash (unit: wt. \%)

\begin{tabular}{|c|c|c|c|c|c|c|c|c|c|c|c|c|c|}
\hline Sample & $\mathbf{C}$ & $\mathbf{O}$ & $\mathbf{S i}$ & $\mathbf{S}$ & $\mathbf{P}$ & $\mathrm{Ca}$ & Al & $\mathbf{F e}$ & Mg & Zn & As & $\mathbf{W}$ & Ge \\
\hline WL 1-1 & 14.01 & 32.67 & 13.17 & 6.33 & 0.21 & 14.0 & 4.59 & 7.82 & 1.3 & 0.15 & 1.4 & 0.18 & 0.043 \\
\hline WL 1-2 & 14.54 & 33.2 & 12.23 & 6.82 & 0.33 & 14.91 & 4.1 & 7.5 & 1.1 & 0.26 & 1.57 & 0.2 & 0.048 \\
\hline WL 1-3 & 15.22 & 33.71 & 13.58 & 5.5 & 0.37 & 14.64 & 3.94 & 8.32 & 1.42 & 0.22 & 1.3 & 0.24 & 0.054 \\
\hline WL 1-4 & 15.3 & 33.54 & 13.53 & 5.7 & 0.26 & 14.21 & 4.47 & 8.12 & 1.2 & 0.15 & 1.32 & 0.18 & 0.047 \\
\hline WL 2-1 & 14.54 & 32.9 & 12.2 & 5.89 & 0.51 & 13.75 & 4.97 & 8.38 & 1.41 & 0.25 & 1.32 & 0.21 & 0.053 \\
\hline WL 2-2 & 14.1 & 33.12 & 11.3 & 6.59 & 0.32 & 14.1 & 5.36 & 8.92 & 1.36 & 0.2 & 1.41 & 0.25 & 0.057 \\
\hline WL 2-3 & 13.48 & 33.88 & 11.82 & 6.57 & 0.44 & 14.25 & 4.65 & 8.26 & 1.47 & 0.3 & 1.48 & 0.25 & 0.051 \\
\hline WL 2-4 & 14.95 & 32.13 & 12.54 & 5.21 & 0.58 & 14.1 & 5.14 & 8.7 & 1.2 & 0.21 & 1.39 & 0.2 & 0.049 \\
\hline WL 3-1 & 16.59 & 33.37 & 10.17 & 4.75 & 0.21 & 14.42 & 4.59 & 8.61 & 1.12 & 0.15 & 1.35 & 0.24 & 0.047 \\
\hline WL 3-2 & 17.03 & 34.2 & 11.23 & 4.2 & 0.33 & 14.91 & 4.1 & 8.5 & 1.1 & 0.26 & 1.57 & 0.37 & 0.055 \\
\hline WL 3-3 & 16.22 & 34.81 & 10.58 & 5.5 & 0.37 & 14.64 & 3.94 & 8.32 & 1.42 & 0.22 & 1.3 & 0.3 & 0.043 \\
\hline WL 3-4 & 17.3 & 33.54 & 10.53 & 3.7 & 0.26 & 14.21 & 4.47 & 8.12 & 1.2 & 0.15 & 1.41 & 0.28 & 0.058 \\
\hline WL 4-1 & 15.42 & 34.41 & 12.5 & 3.71 & 0.3 & 15.2 & 5.8 & 4.65 & 0.84 & 0.09 & 1.65 & 0.3 & 0.051 \\
\hline WL 4-2 & 14.83 & 34.8 & 11.82 & 3.2 & 0.21 & 14.8 & 6.77 & 4.12 & 0.8 & 0.05 & 1.76 & 0.32 & 0.058 \\
\hline WL 4-3 & 13.72 & 35.17 & 13.23 & 4.31 & 0.17 & 13.1 & 6.26 & 4.45 & 0.74 & 0.05 & 1.42 & 0.28 & 0.044 \\
\hline WL 4-4 & 14.8 & 35.6 & 12.4 & 3.69 & 0.36 & 15.5 & 5.91 & 3.65 & 0.7 & 0.06 & 1.46 & 0.35 & 0.05 \\
\hline
\end{tabular}


Table S3. Removal ratio of As and content of Ge and $\mathrm{W}$ in residue under optimal conditions $\left(550{ }^{\circ} \mathrm{C}\right.$ of reaction temperature, adding $50 \mathrm{wt} . \%$ sulfurizing agent, system pressure of $1 \mathrm{~Pa}$, and $240 \mathrm{~min}$ of reaction time)

\begin{tabular}{|c|c|c|c|}
\hline Samples & Removal ratio of As & Content of Ge & Content of W \\
\hline WL 1-1 & $96 \%$ & 0.75 wt. $\%$ & 0.24 wt. $\%$ \\
\hline WL 1-2 & $95 \%$ & 0.75 wt. $\%$ & 0.24 wt. $\%$ \\
\hline WL 1-3 & $96 \%$ & 0.77 wt. $\%$ & 0.23 wt. $\%$ \\
\hline WL 1-4 & $96 \%$ & 0.76 wt. $\%$ & 0.24 wt. $\%$ \\
\hline WL 2-1 & $96 \%$ & 0.76 wt. $\%$ & 0.24 wt. $\%$ \\
\hline WL 2-2 & $96 \%$ & 0.76 wt. $\%$ & 0.24 wt. $\%$ \\
\hline WL 2-3 & $95 \%$ & 0.76 wt. $\%$ & 0.25 wt. $\%$ \\
\hline WL 2-4 & $96 \%$ & 0.75 wt. $\%$ & 0.24 wt. $\%$ \\
\hline WL 3-1 & $96 \%$ & 0.76 wt. $\%$ & 0.24 wt. $\%$ \\
\hline WL 3-2 & $95 \%$ & 0.72 wt. $\%$ & 0.22 wt. $\%$ \\
\hline WL 3-3 & $96 \%$ & 0.74 wt. $\%$ & 0.24 wt. $\%$ \\
\hline WL 3-4 & $96 \%$ & 0.75 wt. $\%$ & 0.24 wt. $\%$ \\
\hline WL 4-1 & $94 \%$ & 0.76 wt. $\%$ & 0.24 wt. $\%$ \\
\hline WL 4-2 & $94 \%$ & 0.73 wt. $\%$ & 0.23 wt. $\%$ \\
\hline WL 4-3 & $96 \%$ & 0.74 wt. $\%$ & 0.24 wt. $\%$ \\
\hline WL 4-4 & $96 \%$ & 0.73 wt. $\%$ & 0.23 wt. $\%$ \\
\hline
\end{tabular}


Table S4. Chemical components of residues from coal fly ash (WL1-1, WL1-2..., WL4-4) (unit: wt. \%)

\begin{tabular}{|c|c|c|c|c|c|c|c|c|c|c|c|c|c|}
\hline Sample & C & $\mathbf{O}$ & $\mathbf{S i}$ & $\mathbf{S}$ & $\mathbf{P}$ & $\mathrm{Ca}$ & Al & $\mathrm{Fe}$ & Mg & Zn & As & $\mathbf{W}$ & Ge \\
\hline WL 1-1 & 5.89 & 30.84 & 14.19 & 0.09 & 0.42 & 22.72 & 6.32 & 13.3 & 1.5 & 0.05 & 0.06 & 0.24 & 0.75 \\
\hline WL 1-2 & 5.32 & 30.11 & 14.66 & 0.08 & 0.34 & 23.64 & 6.4 & 13.83 & 1.43 & 0.04 & 0.08 & 0.24 & 0.75 \\
\hline WL 1-3 & 6.65 & 31.23 & 14.01 & 0.08 & 0.4 & 23.01 & 5.76 & 14.56 & 1.74 & 0.08 & 0.05 & 0.23 & 0.77 \\
\hline WL 1-4 & 6.7 & 31.54 & 13.75 & 0.06 & 0.31 & 22.9 & 5.9 & 14.49 & 1.79 & 0.08 & 0.06 & 0.24 & 0.76 \\
\hline WL 2-1 & 6.41 & 31.23 & 13.65 & 0.11 & 0.34 & 20.82 & 6.78 & 14.05 & 1.7 & 0.07 & 0.05 & 0.24 & 0.76 \\
\hline WL 2-2 & 6.12 & 32.58 & 14.46 & 0.1 & 0.27 & 20.4 & 6.43 & 13.88 & 1.51 & 0.05 & 0.06 & 0.24 & 0.76 \\
\hline WL 2-3 & 5.45 & 32.79 & 14.9 & 0.1 & 0.33 & 21.36 & 6.04 & 13.2 & 1.27 & 0.06 & 0.07 & 0.25 & 0.76 \\
\hline WL 2-4 & 6.79 & 31.9 & 13.98 & 0.12 & 0.3 & 20.51 & 6.9 & 14.32 & 1.8 & 0.05 & 0.06 & 0.24 & 0.75 \\
\hline WL 3-1 & 6.2 & 32.7 & 15.7 & 0.08 & 0.27 & 20.65 & 7.75 & 11.88 & 1.7 & 0.08 & 0.05 & 0.24 & 0.76 \\
\hline WL 3-2 & 6.74 & 33.35 & 16.56 & 0.12 & 0.36 & 20.1 & 7.11 & 11 & 1.4 & 0.09 & 0.08 & 0.22 & 0.72 \\
\hline WL 3-3 & 6.45 & 33.2 & 16.7 & 0.1 & 0.33 & 20.83 & 6.32 & 11.9 & 1.72 & 0.05 & 0.05 & 0.24 & 0.74 \\
\hline WL 3-4 & 6.97 & 33.38 & 16.41 & 0.14 & 0.36 & 20.3 & 7.6 & 10.78 & 1.95 & 0.08 & 0.06 & 0.24 & 0.75 \\
\hline WL 4-1 & 5.91 & 34.8 & 17.57 & 0.07 & 0.23 & 18.8 & 7.05 & 10.4 & 1.2 & 0.13 & 0.10 & 0.24 & 0.76 \\
\hline WL 4-2 & 5.32 & 34.21 & 16.47 & 0.05 & 0.3 & 18.3 & 7.4 & 10.7 & 1.43 & 0.17 & 0.11 & 0.23 & 0.73 \\
\hline WL 4-3 & 4.7 & 33.67 & 17.94 & 0.08 & 0.47 & 19.7 & 7.1 & 11.52 & 1.31 & 0.16 & 0.06 & 0.24 & 0.74 \\
\hline WL 4-4 & 5.51 & 34.53 & 17.1 & 0.05 & 0.3 & 18.29 & 6.85 & 10.7 & 1.45 & 0.17 & 0.06 & 0.23 & 0.73 \\
\hline
\end{tabular}


Table S5. Mechanism functions of kinetics and fitting degree for the vacuum reaction

\begin{tabular}{|c|c|c|c|c|}
\hline Mark & Function & Mechanism & Integral & Fitting degree \\
\hline$\rightarrow-$ & Parabolic & One-dimensional diffusion & $\alpha^{2}$ & \\
\hline$\rightarrow$ & Jander & Two-dimensional diffusion & $\alpha+(1-\alpha) \ln (1-\alpha)$ & 0.988 \\
\hline$\rightarrow-$ & Jander & $\begin{array}{l}\text { Two-dimensional diffusion, } 2 \mathrm{D} \text {, } \\
\mathrm{n}=2\end{array}$ & $\left(1-(1-\alpha)^{1 / 3}\right)^{2}$ & 0.985 \\
\hline & G-B model & $\begin{array}{l}\text { Three-dimensional diffusion, 3D, } \\
\text { D4 }\end{array}$ & $1-2 / 3 \alpha-(1-\alpha)^{2 / 3}$ & 0.987 \\
\hline & Anti-Jander & three-dimensional diffusion, 3D & $\left((1-\alpha)^{-1 / 3}-1\right)^{2}$ & 0.980 \\
\hline$\rightarrow-$ & Mample & Random nucleation growth, $\mathrm{n}=1$ & $1-(1-\alpha)^{1}$ & 0.988 \\
\hline & Contraction cylinder & $\begin{array}{l}\text { Phase boundary reaction, } \\
\text { cylindrical symmetry }\end{array}$ & $1-(1-\alpha)^{1 / 2}$ & 0.984 \\
\hline & Contraction sphere & $\begin{array}{l}\text { Phase boundary reaction, sphere } \\
\text { symmetry }\end{array}$ & $1-(1-\alpha)^{1 / 3}$ & 0.983 \\
\hline & Reaction series & $\mathrm{n}=1 / 4$ & $1-(1-\alpha)^{0.25}$ & 0.982 \\
\hline & Mample & $\begin{array}{l}\text { Random nucleation growth, } \mathrm{n}=1 \text {, } \\
\mathrm{m}=1\end{array}$ & $-\ln (1-\alpha)$ & 0.980 \\
\hline & Avrami-Erofeev & $\begin{array}{l}\text { Random nucleation growth, } \\
n=1 / 2, m=2\end{array}$ & $-\ln (1-\alpha)^{0.5}$ & 0.973 \\
\hline & Avrami-Erofeev & $\begin{array}{l}\text { Random nucleation growth, } \\
n=1 / 3, m=3\end{array}$ & $(-\ln (1-\alpha))^{1 / 3}$ & 0.959 \\
\hline- & Avrami-Erofeev & $\begin{array}{l}\text { Random nucleation growth, } \\
n=1 / 4, m=4\end{array}$ & $(-\ln (1-\alpha))^{0.25}$ & 0.931 \\
\hline & Avrami-Erofeev & $\begin{array}{l}\text { Random nucleation growth, } \\
n=3 / 2\end{array}$ & $(-\ln (1-\alpha))^{1.5}$ & 0.982 \\
\hline$\rightarrow-$ & Avrami-Erofeev & Random nucleation growth, $\mathrm{n}=2$ & $(-\ln (1-\alpha))^{2}$ & 0.983 \\
\hline$-\infty$ & Avrami-Erofeev & Random nucleation growth, $\mathrm{n}=3$ & $(-\ln (1-\alpha))^{3}$ & 0.983 \\
\hline-- & Avrami-Erofeev & Random nucleation growth, $\mathrm{n}=4$ & $(-\ln (1-\alpha))^{4}$ & 0.984 \\
\hline$\rightarrow$ & Reaction series & $\mathrm{n}=2$ & $1-(1-\alpha)^{2}$ & 0.995 \\
\hline$\rightarrow-$ & Secondary reaction & Chemical reaction, F2 & $(1-\alpha)-1$ & 0.594 \\
\hline-- & Chemical reaction & Chemical reaction & $(1-\alpha)^{-0.5}$ & 0.038 \\
\hline$\rightarrow$ & Reaction series & Chemical reaction & $(1-\alpha)^{-1}-1$ & 0.97 \\
\hline$\longrightarrow-$ & Chemical reaction & Tertiary reaction & $(1-\alpha)-2$ & 0.78 \\
\hline
\end{tabular}

